\title{
Orientation Dependence of Step Stiffness: Failure of SOS and Ising Models to Describe Experimental Data
}

\author{
Sabine Dieluweit, Harald Ibach and Margret Giesen* \\ Institut für Schichten und Grenzflächen, ISG 3, Forschungszentrum Jülich, D 52425 Jülich, Germany \\ T. L. Einstein \\ Department of Physics, University of Maryland, College Park, Maryland 20742-4111, USA
}

(Dated: March 6, 2019)

\begin{abstract}
We have investigated the step stiffness on $\mathrm{Cu}(001)$ surfaces as a function of step orientation by two independent methods at several temperatures near $300 \mathrm{~K}$. Both sets of data agree well and show a substantial dependence of the stiffness on the angle of orientation. With the exception of steps oriented along $\langle 110\rangle$, the experimental stiffness is significantly larger than the stiffness calculated within the solid-on-solid (SOS) model and the Ising-model, even if next nearest-neighbor interactions are taken into account. Our results have considerable consequences for the understanding and for the theoretical modeling of equilibrium and growth phenomena, such as step meandering instabilities.
\end{abstract}

PACS numbers: 05.70.Np,68.35.Md,68.37.Ef,81.10.Aj

Self-assembly of nanostructures is a key route on the roadmap of device technology at ever-shrinking lengths. Several methods for self-assembly of nanostructures via kinetics, kinetic instabilities, or as equilibrium phenomena are associated with the properties of steps on solid surfaces [1]. In the context of nanostructures the step stiffness $\tilde{\beta}$-which describes the resistance of a step to meandering - is one of the key parameters in the widely applicable step continuum model [2]. As the free energy cost per length (along the macroscopic edge direction) for elongating the microscopic length of a step due to fluctuations, $\tilde{\beta}$ plays a central role in virtually all properties involving step excitations. For steps in equilibrium, the experimental and theoretical work focussed on spatial and temporal fluctuations and the repulsive or attractive interactions between fluctuating steps $[2,3]$. When steps represent a growth front, stable step-flow growth occurs due to the presence of an Ehrlich-Schwoebel (ES) barrier $[4,5]$ which hinders interlayer diffusion. This barrier, however, can also give rise to a step meandering instability: Bales and Zangwill [6] showed that this form of instability selects a fastest-growing mode of meandering, which determines the morphology of the surface in the long-time limit. More recently it was shown that meandering can also be caused by a kink Ehrlich-Schwoebel barrier (KESE) [7, 8]. Experimentally, a meandering instability was observed on $\mathrm{Cu}(001)$ vicinals by Maroutian et al. [9]. The existing theories, while accounting qualitatively for the effect, fail to give such quantitative aspects as the temperature-dependence of the dominant wave length [9].

Regardless of the precise cause of the instability, the forces driving the step into meandering compete against diffusion currents tending to smooth out gradients in the chemical potential $\mu$ along steps. The chemical potential is the product of $\tilde{\beta}$ and the curvature $\kappa$. Thus, it is the stiffness which keeps deviations from the mean direction low. For steps in equilibrium, such as on vicinal surfaces in the absence of growth, the stiffness determines the magnitude of the equilibrium fluctuations of steps and thereby the fluctuations in the terrace-width distribution.

Unlike the surface stiffness, the step stiffness has a defined value for any orientation since a step is thermodynamically rough above temperature $T=0$. Present analytical theories of the meandering instabilities merely consider an angle independent ("isotropic") stiffness [813 and estimate $\tilde{\beta}(\theta)$ as its value for a step orientation along the direction $\theta=0$ of close packing (cf. Eq. (3) below). In Monte-Carlo simulations (e.g. Ref. [8]), where the modeling of the step meandering is based on the solidon-solid (SOS) model [14], a particular angular dependence of the stiffness is automatically built in by considering an angle dependent line tension (step free energy per length) $\beta(\theta)$ ( since $\left.\tilde{\beta}(\theta)=\beta(\theta)+\beta^{\prime \prime}(\theta)\right)$.

In this Letter we show that not only the standard assumption of isotropic stiffness but even the angulardependent step stiffness of the SOS or Ising model [15] is far from reality for the much-studied $[2,3] \mathrm{Cu}(001)$ surface, typical of late-transition/noble metal surfaces and on which the meandering instability was observed [9]: Our experimentally determined stiffness varies by several orders of magnitude for orientations close to $\langle 110\rangle$, in agreement with the SOS and Ising models. For larger angles, however, the experimental stiffness is about quadruple the value calculated in the two models $[16,17]$. As we will argue, this deviation cannot be accounted for by including next-nearest-neighbor interactions. Rather it must be attributed to many-body interactions such as kink-kink interactions and/or effective corner energies.

Our step-stiffness data come from two independent experiments. One set of data is obtained by measuring the step-step distance correlation function for steps which are misoriented by an azimuthal angle $\theta$ from the high-symmetry, close-packed $\langle 110\rangle$ direction and there- 
fore contain forced kinks. The second set is obtained by analyzing equilibrium shapes of two-dimensional islands $[18,19]$. Here $\theta$ denotes the angle between the local island-edge orientation and the $\langle 110\rangle$ direction (so that $\tan \theta$ corresponds to the local slope) rather than (as in Ref. [18], e.g.) the angle denoting the position on the perimeter [20].

The experiments were performed in a standard ultrahigh vacuum (UHV) chamber equipped with a variabletemperature microscope $[21,22]$ with high thermal drift stability. For the experiments on the step-step correlation function we used copper surfaces with Miller indices $(5,8,90)$. Hence, the surfaces are tilted relative to the (001) surface by the polar angle $\phi=5.98^{\circ}$. The resulting monatomic steps on the surface are rotated with respect to the close-packed $\langle 110\rangle$ direction by a nominal angle $\theta=12.99^{\circ}$, which corresponds to a density 0.23 of geometric kinks. The accuracy of orientation is naturally limited by the mosaic structure of the single crystal. Crystal cleaning procedures followed established recipes [19] after which the sample surfaces revealed clean parallel steps, which, however, merely on the average display the nominal rotation angle of $12.99^{\circ}$ of the $(5,8,90)$ surface. The misalignment $\theta$ of the steps with respect to the $\langle 110\rangle$-direction varied locally between $0^{\circ}$ and $45^{\circ}$. The size of the local area of constant angle was of order $10^{4} \mathrm{~nm}^{2}$. Fig. 1 shows an STM image of the $\mathrm{Cu}(5,8,90)$ surface at $301 \mathrm{~K}$ with $\theta=7.13^{\circ}$, i.e. with an average concentration 0.13 of forced kinks. The mean value of $\theta$ of the steps was determined by averaging over all steps in the image. As is obvious from Fig. 1, the $\langle 110\rangle$ direction (black solid arrow in Fig. 1) is easily defined in an STM image by the straight step segments between individual, monatomic kinks.

Spatial fluctuations of steps can be analyzed via a step correlation function $G(y)[25]$

$$
G(y)=\left\langle\left[x\left(y+y^{\prime}\right)-x\left(y^{\prime}\right)\right]^{2}\right\rangle=\frac{k_{B} T}{\tilde{\beta} a_{\|}}|y| a_{\|},
$$

where $y$ and $y^{\prime}$ are coordinates parallel to the mean direction along which the step runs (see Fig. 1) [26], while $a_{\|}$is the atomic length unit (along $\langle 110\rangle$ ). We have measured the spatial correlation function $G(y)$ for various angles $\theta$ on the $\mathrm{Cu}(5,8,90)$ surface at temperatures between 293 and $328 \mathrm{~K}$. For the highest temperature and larger angles $\theta$, the plot of $G(y)$ showed a small influence of the time dependence $[23,24]$ of the fluctuations by the curving of $G(y)$ at small $y$. The slope in Eq. (1) was determined for larger $y$ where $G(y)$ was a linear. The resulting values for the dimensionless quantity $k_{B} T / \tilde{\beta} a_{\|}$are plotted in Fig. 2 as gray symbols. The data points represent an average over 100 individual steps with a total step length of 1-2 $\mu \mathrm{m}$ each. Included as stars are data at $\theta=0^{\circ}$ taken from previous work of our group for $\mathrm{Cu}(11 \mathrm{n})$ surfaces, $n=13$ and 19 , for which $k_{B} T / \tilde{\beta}(0) a_{\|}=0.019$ and 0.0129 , respectively $[24,27,28]$.

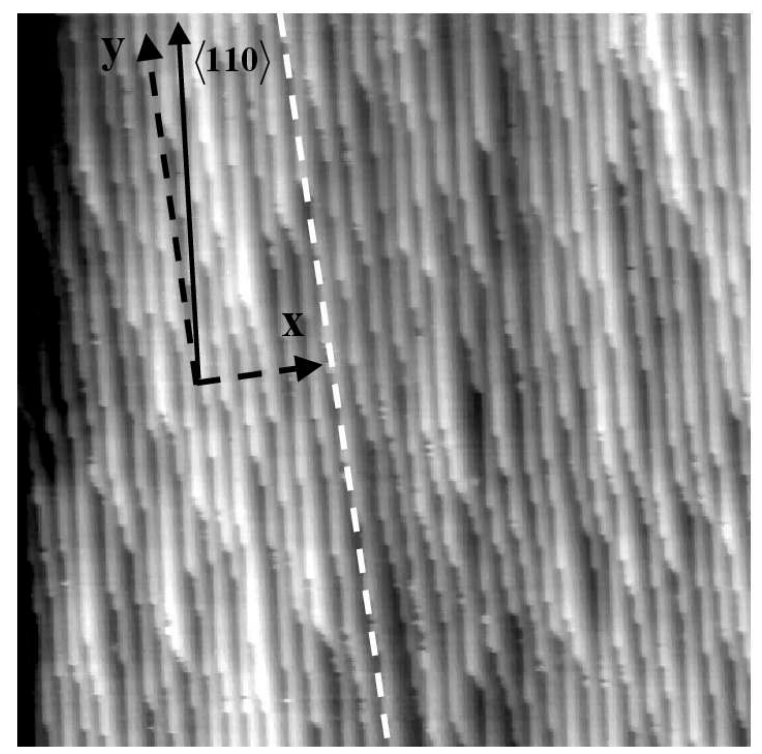

FIG. 1: STM image of a surface region on $\mathrm{Cu}(5,8,90)$ where on the average the steps run $7.13^{\circ}$ off the atomically dense $\langle 110\rangle$ direction. The scan width is $24.3 \mathrm{~nm}$.

The step stiffness $\tilde{\beta}$ can also be determined experimentally by analyzing equilibrium shapes of islands [19]. Islands in equilibrium have a well-defined chemical potential which is the same all along the perimeter of an island. The chemical potential of the island is proportional to the product of the curvature $\kappa(\theta)$ of the island perimeter and the step stiffness $\tilde{\beta}(\theta)$ (via the Gibbs-Thomson equation).

For islands in equilibrium, as shown previously [19], the chemical potential can likewise be equated to the free energy per length $\beta_{0}$ of the densely packed steps oriented along $\langle 110\rangle$ divided by the distance of this step segment from the center of the island $r_{0}$. Then one obtains

$$
\tilde{\beta}(\theta)=\frac{\beta_{0}}{r_{0} \kappa(\theta)} .
$$

Since the absolute number for the step free energy $\beta_{0}$ for $\mathrm{Cu}(001)$ is known from experiment, $\beta_{0}=220 \mathrm{meV} a_{\|}^{-1}$ $[19,29]$, Eq. (2) enables the calculation of the orientation dependence of $k_{B} T / \tilde{\beta}(\theta) a_{\|}$from the equilibrium island shape. Data were extracted from experiment by averaging over more than a thousand individual island shapes which were measured at temperatures between 287 and $313 \mathrm{~K}$. The temperature dependence of the equilibrium shapes is small in the range of $\theta$ of interest here. Using the mean shapes obtained for each temperature we have calculated the curvature as a function of the angle $\theta$ and furthermore, $k_{B} T / \tilde{\beta}(\theta) a_{\|}$following Eq. (2). The temperature-averaged results are plotted in Fig. 2 as solid diamonds. They agree quite well with the data from the correlation function $G(y)$, except for $\theta=0$ where it ap- 


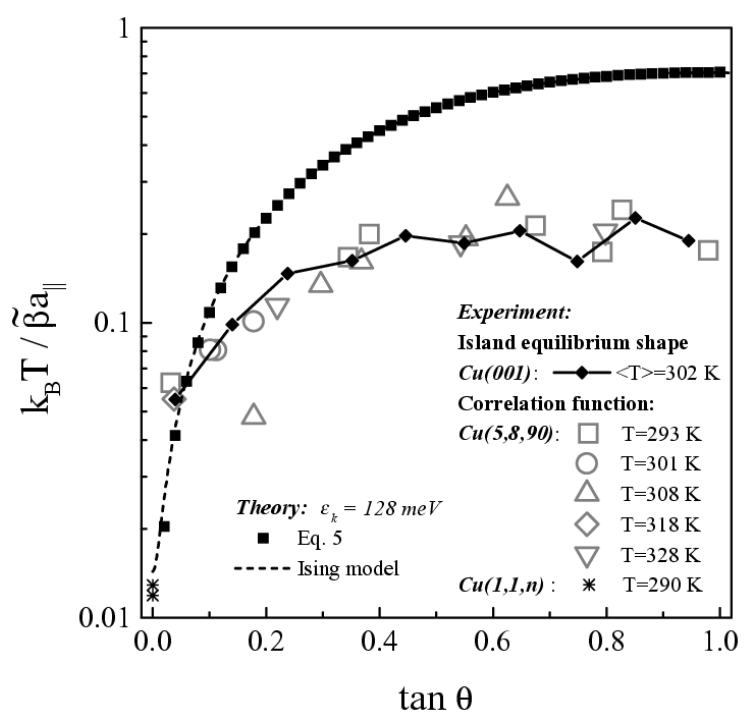

FIG. 2: Dimensionless inverse stiffness $k_{B} T / \tilde{\beta}(\theta) a_{\|}$measured via spatial step fluctuations (gray symbols) and via island equilibrium shapes (solid diamonds). Full squares represent Eq. (6) and the dashed line is calculated from the temperature-dependent analytical solution of the island equilibrium shape in the nearest-neighbor Ising model.

pears as if $k_{B} T / \tilde{\beta}(0) a_{\|}$obtained from the island equilibrium shapes would be significantly higher. This is, however, an artifact of the island shape analysis. Technically one cannot determine the rapidly changing curvature of the island equilibrium shape near the $\langle 110\rangle$ direction.

The rapid descent of $k_{B} T / \tilde{\beta}(\theta) a_{\|}$close to $\theta=0$ is a real effect. It can be shown that at $\theta=0$ and for moderate temperatures, $k_{B} T / \tilde{\beta}(0) a_{\|}$equals twice the concentration of thermally excited kinks [25]:

$$
k_{B} T / \tilde{\beta}(0) a_{\|}=2\left(a_{\perp} / a_{\|}\right)^{2} e^{-\epsilon_{k} / k_{B} T},
$$

where $\epsilon_{k}$ is the kink energy. Since the unit spacing $a_{\perp}$ between $\langle 110\rangle$ rows is the same as $a_{\|}$for an (001) fcc surface, we do not distinguish between them henceforth, calling both $a_{1}$. Eq. (3) is model independent and exact if $z \equiv \exp \left(-\epsilon_{k} / k_{B} T\right) \ll 1$.

In order to compare our experimental data for $k_{B} T / \tilde{\beta}(\theta) a_{1}$ with theory, we make use of explicit exact expressions for $\beta(\theta)$ for the nearest-neighbor Ising and SOS models $[16,17]$, from which we obtain $\tilde{\beta}(\theta)$ as $\beta(\theta)+\beta^{\prime \prime}(\theta)$. While the general expression can be readily handled numerically, $T \ll \epsilon_{k} / k_{B}$ in the present problem, permitting an expansion in $z$ (which is of order $6 \times 10^{-3}$ when we specify $\epsilon_{k}$ below). For the Ising model, Rottman and Wortis [16] found

$$
\begin{array}{r}
\beta(\theta) a_{1} / k_{B} T=\left(\epsilon / k_{B} T\right)(|\cos \theta|+|\sin \theta|) \\
-((|\cos \theta|+|\sin \theta|) \ln (|\cos \theta|+|\sin \theta|)
\end{array}
$$

$$
\begin{array}{r}
-|\cos \theta| \ln (|\cos \theta|)-|\sin \theta| \ln (|\sin \theta|)) \\
-\frac{|\cos \theta|^{3}+|\sin \theta|^{3}}{|\cos \theta||\sin \theta|} z^{2}+O\left(z^{4}\right) .
\end{array}
$$

The energy-like first term in Eq. (4) makes no contribution to $\tilde{\beta}(\theta)$ since it is canceled by its second derivative. We then obtain the following expansion for the stiffness:

$$
\begin{aligned}
k_{B} T / \tilde{\beta}(\theta) a_{1} & =(|\cos \theta|+|\sin \theta|)|\cos \theta||\sin \theta| \\
+2(|\cos \theta| & +|\sin \theta|)^{3}(|\csc \theta||\sec \theta|-1) z^{2}+O\left(z^{4}(5)\right.
\end{aligned}
$$

The first term in Eq. (5) increases gradually but monotonically with $\theta$, while the monotonically-decreasing coefficient of $z^{2}$ is sharply peaked at $\theta=0$. Hence, except near $\theta=0$ (specifically, so long as $\tan \theta \gg z$ ), the $z^{2}$ term in Eq. (5) can be neglected, leaving

$$
\frac{k_{B} T}{\tilde{\beta}(\theta) a_{1}} \approx\left(\tan ^{2} \theta+|\tan \theta|\right)|\cos \theta|^{3}=\frac{\tan ^{2} \theta+|\tan \theta|}{\left(1+\tan ^{2} \theta\right)^{\frac{3}{2}}}
$$

The result in Eq. (6) can be obtained from a combinatorical analysis for the number of ways of arranging the kinks forced by the azimuthal misorientation $\theta[16,30]$. Since we expect $k_{B} T / \tilde{\beta}(\theta) a_{1} \rightarrow 2 z$ as $\theta \rightarrow 0$, the expansion in Eq. (5) evidently fails near this limit.

The temperature independent expression for $k_{B} T / \tilde{\beta}(\theta) a_{1}$ from Eq. (6) is displayed in Fig. 2 as solid squares. With the help of Eq. (2) $k_{B} T / \tilde{\beta}(\theta) a_{1}$ can also be calculated for arbitrary temperatures from the analytical solution of the island equilibrium shape within the Ising model $[17,19]$. The result is displayed as a dashed line in Fig. 2. The Ising parameter representing the kink energy as well as the step energy per atom was chosen as $\epsilon_{k}=128 \mathrm{meV}$ [24, 27].

While experimental data and theory agree well for small $\theta$, they disagree substantially for larger $\theta$. The agreement for small $\theta$ is due to the fact that eq. (4) is independent of any assumption other than $z \ll 1$. The reason for the deviation at larger $\theta$ can be understood best by considering the special case $\theta=45^{\circ}$. From Eq. (6) one obtains

$$
k_{B} T / \tilde{\beta}\left(45^{\circ}\right) a_{1}=1 / \sqrt{2} .
$$

This result can be derived stragihtforwardly by considering the spatial correlation function $G(y)$ for a freely meandering steps in the $\langle 100\rangle$ direction if overhangs are excluded and the statistical weight factors for microscopic paths take only nearest neighbor broken bonds into account. As overhangs are excluded the result is correct to terms of order $z^{4}$ which is an excellent approximation for the temperatures considered here. The fact that $k_{B} T / \tilde{\beta}(\theta) a_{1}$ is lower than predicted by Eq. (7) must therefore mean that the $\langle 100\rangle$ oriented step $\left(\theta=45^{\circ} ; 100 \%\right.$ kinked) with one kink following another is energetically 
favored over paths which deviate from this orientation. This is in accord with the analysis of island equilibrium shapes [19], which found that the ratio of line tensions $\beta_{\langle 100\rangle} / \beta_{\langle 110\rangle}$ at $T=0 \mathrm{~K}$ is 1.24 rather than $\sqrt{2}$ as predicted in the Ising model.

We note that diagonal, next-nearest neighbor interactions characterized by $\epsilon_{n n}$ cannot be the sole explanation. It is straightforward to show that the experimentally observed line tensions for the $\langle 100\rangle$ and $\langle 110\rangle$ directions cannot be fit simultaneously with the kink energy to a model with nnn-pair-potentials. As for the temperature dependence, nnn-interactions were considered, e.g., in the Akutsus' study of $\mathrm{Si}(001)$ [31]. To leading order, their expansion factor $\exp \left(-\epsilon_{k} / k_{B} T\right)$ becomes $\exp \left(-\left(\epsilon_{k}+2 \epsilon_{n n} / k_{B} T\right)\right.$. However, this change only affects the negligible $z^{2}$ term; there is no change to Eq. (6). Thus, the lower line tension of steps at larger angles $\theta$ and, in particular, that of a $\langle 100\rangle$-oriented step $\left(\theta=45^{\circ}\right)$ and the lower corresponding spatial step fluctuations can therefore not be described by a nnn-pair potential. Rather they must be attributed to many body interactions. Such many body contributions might exist in the form of (attractive) kink-kink interactions and/or (negative) corner energies involved in the formation of kinks in a $\langle 100\rangle$-step. These interactions would keep a meandering step with a mean orientation along $\langle 100\rangle$ close to the central path which is also microscopically a $\langle 100\rangle$-direction and in which kinks follow immediately one after another. Spatial fluctuations would therefore be smaller than in the Ising model. Models which might produce such behavior are under investigation [32].

We finally comment on the consequences of the strong angle dependence of the stiffness on the step meandering instability [6-13]. Under plausible assumptions, the most unstable, fastest growing mode $\lambda_{u} \propto \tilde{\beta}^{1 / 2}$ in a linear stability analysis. Using then Eq. (3) for the calculation of $\lambda_{u}$ not only overestimates the effective stiffness of the technically always slightly misoriented steps, it also introduces an unrealistic temperature dependence. A very recent theoretical study [33] of the meandering instability of $\mathrm{Cu}(001)$ did consider a step stiffness with angular dependence, but with behavior so different [34] from Eq. (6) to preclude useful comparison. Recognition of the remarkable angular dependence of the stiffness is a crucial ingredient for attempts to account for the meandering instabilities [9] and other statistical properties of vicinals and of single-layer islands.

\section{Acknowledgment}

We acknowledge the high-accuracy sample preparation by Udo Linke. Furthermore, we have benefited from helpful discussion with Joachim Krug. This work was partially supported by the Fond der Chemischen Industrie, Germany. TLE thanks the Humboldt Foundation for generous support under a U.S. Senior Scientist Award and is also grateful for funding from NSF-MRSEC, grant DMR 00-80008.

* Corresponding author

Email: m.giesen@fz-juelich.de

Internet: http://www.fz-juelich.de/isg/isg3/Giesen/aggiesen1.htm

* Electronic address: m.giesen@fz-juelich.de\%\\ Internet:http://www.fz-juelich.de/isg/isg3/ Giesen/ag-giesen $1 . \mathrm{htm}$

[1] C. Teichert, Phys. Rep. 365, 335 (2002).

[2] H.-C. Jeong and E. D. Williams, Surf. Sci. Rep. 34, 171 (1999).

[3] M. Giesen, Prog. Surf. Sci. 68, 1 (2001).

[4] G. Ehrlich and F. G. Hudda, J. Chem. Phys. 44, 1039 (1966).

[5] R. L. Schwoebel and E. J. Shipsey, J. Appl. Phys. 37, 3682 (1966).

[6] G. S. Bales and A. Zangwill, Phys. Rev. B 41, 5500 (1990).

[7] O. Pierre-Louis, M. R. D’Orsogna, and T. L. Einstein, Phys. Rev. Lett. 82, 3661 (1999).

[8] J. Kallunki, J. Krug, and M. Kotrla, Phys. Rev. B 65, 205411 (2002).

[9] T. Maroutian, L. Douillard, and H.-J. Ernst, Phys. Rev. Lett. 83, 4353 (1999); Phys. Rev. B 64, 165401 (2001).

[10] O. Pierre-Louis, C. Misbah, Y. Saito, et al., Phys. Rev. Lett. 80, 4221 (1998).

[11] F. Gillet, O. Pierre-Louis, and C. Misbah, Eur. Phys. J. B 18, 519 (2000).

[12] J. Kallunki and J. Krug, Phys. Rev. E 62, 6229 (2000).

[13] O. Pierre-Louis, Phys. Rev. Lett. 87, 106104 (2001).

[14] W. Kossel, Nachr. Ges. Wiss. Göttingen 135 (1927).

[15] For the much-studied semiconductor surface $\mathrm{Si}(111)$, the Ising model did prove useful in characterizing $\beta(\theta)$; see E. D. Williams, R. J. Phaneuf, J. Wei, et al., Surf. Sci. 294, 219 (1993); 310, 451 (E) (1994).

[16] C. Rottman and M. Wortis, Phys. Rev. B 24, 6274 (1981).

[17] J. E. Avron et al., J. Phys. A 15, L81 (1982); R. K. P. Zia and J. E. Avron, Phys. Rev. B 25, 2042 (1982).

[18] G. Schulze-Icking-Konert, M. Giesen, and H. Ibach, Phys. Rev. Lett. 83, 3880 (1999).

[19] M. Giesen, C. Steimer, and H. Ibach, Surf. Sci. 471, 80 (2001).

[20] That angle is $\theta+\tan ^{-1}\left(\beta^{\prime}(\theta) / \beta(\theta)\right)$, as shown by S. Kodambaka, S. V. Khare, V. Petrova, A. Vailionis, I. Petrov, and J. E. Greene, Surf. Sci. 513, 468 (2002).

[21] K. Besocke, Surf. Sci. 181, 145 (1987).

[22] J. Frohn, J. F. Wolf, K. Besocke, et al., Rev. Sci. Instrum. 60, 1200 (1989).

[23] M. Giesen-Seibert, R. Jentjens, M. Poensgen, et al., Phys. Rev. Lett. 71, 3521 (1993); 73, 911 (E) (1994).

[24] M. Giesen-Seibert and H. Ibach, Surf. Sci. 316, 205 (1994).

[25] N. C. Bartelt, T. L. Einstein, and E. D. Williams, Surf. Sci. 276, 308 (1992). 
[26] P. Nozières, in Solids Far from Equilibrium, edited by C. Godrèche (Cambridge University Press, Cambridge, 1991), p. 1.

[27] M. Giesen-Seibert, F. Schmitz, R. Jentjens, et al., Surf. Sci. 329, 47 (1995).

[28] Also, consistent values at room $T$ for $\mathrm{Cu}(1,1,11)$ and (810) were reported by J. C. Girard, S. Gauthier, S. Rousset, and J. Klein, Microsc. Microanal. Microstruct. 4, 489 (1993).

[29] C. Steimer, M. Giesen, L. Verheij, et al., Phys. Rev. B 64, 085416 (2001).
[30] J. W. Cahn and R. Kikuchi, J. Phys. Chem. Solids 20, 94 (1961).

[31] N. Akutsu and Y. Akutsu, Surf. Sci. 376, 92 (1997).

[32] H. Gebremariam, F. Szalma, and T. L. Einstein, unpublished.

[33] H. Emmerich, Phys. Rev. B 65, 233406 (2002).

[34] Specifically, from use of a Lennard-Jones potential, Ref. [33] claims that the minimum stiffness occurs for steps in the $\langle 130\rangle$-direction of $\mathrm{Cu}(001)$ and has simple sinusoidal variation proportional to "crystalline anisotropy." 